\title{
Association between the nicotinamide adenine dinucleotide phosphate oxidase p22phox gene -A930G polymorphism and intracerebral hemorrhage
}

\author{
YUAN ZHOU $^{1}$ and YING-CHUN ZHAO ${ }^{2}$ \\ ${ }^{1}$ Department of Neurology, Jiuting Hospital; ${ }^{2}$ Department of Neurology, Songjiang Central Hospital, \\ Shanghai 201600, P.R. China
}

Received December 24, 2013; Accepted September 24, 2014

DOI: $10.3892 / \mathrm{mmr} .2015 .3154$

\begin{abstract}
The present study aimed to evaluate whether the -A930G polymorphism of the nicotinamide adenine dinucleotide phosphate $[\mathrm{NAD}(\mathrm{P}) \mathrm{H}]$ oxidase p22phox gene is involved in intracerebral hemorrhage ( $\mathrm{ICH})$ in the Chinese Han population. In the present case-control investigation, the subjects included 118 patients with ICH and 147 healthy controls. The -A930G polymorphism was determined using polymerase chain reaction and restriction fragment length polymorphism. Furthermore, the correlation between the -A930G gene polymorphism and $\mathrm{ICH}$ was evaluated using statistical analyses. The distribution of p22phox -A930G genotypes differed significantly between the two groups $(\mathrm{P}=0.003)$, with the AA, AG and GG genotype frequencies being 61.9, 29.3 and $8.8 \%$ in the control group and $40.7,45.8$ and $13.6 \%$ in the $\mathrm{ICH}$ group, respectively. The $\mathrm{G}$ allele frequency was significantly higher in patients with ICH compared with healthy controls (36.4 vs. $23.5 \%$; $\mathrm{P}<0.05$ ), however, the opposite was observed in the frequency of the A allele (63.6 vs. $76.5 \%$; $\mathrm{P}<0.05)$. Binary logistic regression analysis revealed that genetic mutations of the $\mathrm{p} 22$ phox -A930G gene were independent risk factors of ICH (odds ratio, 2.196; 95\% confidence interval, 1.003-4.586; $\mathrm{P}=0.009)$. In addition, certain conventional factors were associated with increased risk of $\mathrm{ICH}$, including elevated blood pressure, increased levels of glucose and triglycerides in the blood, a history of hypertension and smoking. The -A930G polymorphism of the p22phox gene may affect the susceptibility to $\mathrm{ICH}$ and certain haplotypes of the gene may be associated with a higher susceptibility to ICH.
\end{abstract}

Correspondence to: Dr Ying-chun Zhao, Department of Neurology, Songjiang Central Hospital, 155 Jiuting Road, Shanghai 201600, P.R. China

E-mail: zhaoyingchun9077@163.com

Key words: A930G polymorphism, intracerebral hemorrhage, Chinese Han

\section{Introduction}

Cerebral hemorrhage, also termed intracerebral hemorrhage (ICH), is a subtype of intracranial hemorrhage occurring within the brain tissue, which can be caused by brain trauma or can occur spontaneously in hemorrhagic stroke (1). ICH is a common fatal subtype of stroke and can cause mortality or severe disability $(1,2)$. ICH causes mortality in $>30,000$ individuals annually in the United States (3). Following ICH, the resulting hematoma within the brain parenchyma triggers a series of adverse events, which lead to secondary insults and severe neurological deficits (4). Epidemiological studies have identified differences in the incidence of cerebral hemorrhage between different ethnicities, with African American and Japanese individuals having an incidence rate twice and four times that of Caucasians, respectively (5). Although hypertensive arteriolosclerosis and cerebral amyloid angiopathy are risk factors that contribute to the majority of ICH cases, several genetic risk factors have been identified (6). Characterization of these genetic variations may enable improvements in the prognosis and prevention of $\mathrm{ICH}$.

Reactive oxygen species (ROS), including superoxide, hydrogen peroxide and hydroxyl radicals, are generated by the partial reduction of oxygen (7). ROS in the vasculature are produced by several enzymatic systems, including xanthine oxidase, nitric oxide synthases and nicotinamide adenine dinucleotide phosphate $[\mathrm{NAD}(\mathrm{P}) \mathrm{H}]$ oxidases. Previous studies have indicated that NAD $(\mathrm{P}) \mathrm{H}$ oxidases are major factors involved in the aberrant production of ROS in the vasculature $(7,8)$. Cumulative evidence has demonstrated that ROS are essential in the pathogenesis of various types of cardiovascular disease, including stroke and atherosclerotic lesions (9). ROS-mediated oxidative modification of phospholipids can damage endothelial cells and induce the expression of adhesion molecules, which leads to attachment of monocytes, T lymphocytes and macrophages to the endothelial cells (10). It is hypothesized that the diversity of novel oxidative modification to lipids and proteins mediated by ROS is associated with foam cell formation, the development of atherosclerotic lesions and ultimately a fibrous plaque that protrudes into the arterial lumen (11). The formation and release of thrombi may occlude vessels, which causes 
cardiovascular diseases, including hypertension, congestive heart failure, ICH and stoke (12).

There is accumulating evidence that the -A930G polymorphism of the p22phox subunit of $\mathrm{NAD}(\mathrm{P}) \mathrm{H}$ oxidases is associated with ICH (13). Membrane-associated NAD $(\mathrm{P}) \mathrm{H}$ oxidases are the main enzymes responsible for ROS generation in neutrophil granulocytes and the vasculature, which may be important in the pathogenesis of ischemic injury and atherosclerosis (14). The $\mathrm{NAD}(\mathrm{P}) \mathrm{H}$ oxidase system has been well characterized, comprising of three cytosolic elements, p40phox, p47phox and p67phox, two membrane-associated cytochromes, p22phox and gp91phox and a low molecular weight G-protein (15). Polymorphism of the p22phox gene has been investigated the most of those in the NAD(P)H oxidase system. The gene encoding the p22phox subunit, CYBA, is located on the long arm of chromosome 16 (16q24), spans a length of $8.5 \mathrm{~kb}$ and contains six exons and five introns (16). The -A930G polymorphism is located at the promoter region and the $\mathrm{G}$ allele has increased promoter activity and a higher level of ROS, leading to increased vascular smooth muscle cell proliferation, endothelial dysfunction and the formation of lesions, which can contribute to cerebrovascular disease (13). It has been observed that the $-\mathrm{A} 930 \mathrm{G}$ polymorphism is associated with hypertension. Hypertensive patients with a GG genotype exhibit increased p22phox mRNA and protein levels and enhanced NAD(P)H oxidase activity compared with those with an AA or AG genotype, while no difference is observed in these parameters in healthy subjects (13).

However, it remains unclear whether the -A930G polymorphism of the p22phox gene is associated with $\mathrm{ICH}$. The purpose of the present case-control study was to determine whether the -A930G polymorphism of the NAD $(\mathrm{P}) \mathrm{H}$ oxidase $\mathrm{p} 22$ phox gene is important in Chinese Han patients with $\mathrm{ICH}$, using the method of polymerase chain reaction (PCR), restriction fragment length polymorphism (RFLP) and gene sequencing.

\section{Subjects and methods}

Subjects.Recruitment of individuals for the present case-control study was performed in the Department of Internal Neurology from Shanghai Songjiang Central Hospital (Shanghai, China). All individuals were from the Chinese Han population of Shanghai. The ICH group included 118 patients, confirmed using computed tomography scan and/or magnetic resonance imaging and healthy controls, which included 147 individuals of matched age and gender. Individuals with cardiocerebral injuries, tumors, surgery, external injuries or severe heart, lung, kidney diseases were excluded from the investigation and individuals with a history of stroke or related diseases were also excluded from the control group. The present study complied with the Helsinki declaration and was approved by the Ethics Committee of Shanghai Songjiang Central Hospital. All of the individuals provided informed consent. Blood glucose and blood lipid examinations were performed on all subjects. Previous diagnoses of hypertension and diabetes were also recorded. Hypertension was diagnosed at a blood pressure level of $\geq 140 / 90 \mathrm{mmHg}$. Diabetes was diagnosed at a fasting glucose level of $>7 \mathrm{mmol} / \mathrm{l}$. Individuals with levels of blood cholesterol $\geq 5.2 \mathrm{mmol} / 1$, triglyceride $\geq 2.3 \mathrm{mmol} / 1$, high density lipoprotein (HDL) $\leq 1.8 \mathrm{mmol} / 1$, low density lipoprotein
$(\mathrm{LDL}) \geq 4.9 \mathrm{mmol} / 1$, apolipoprotein (Apo)A $\geq 2.25 \mathrm{~g} / 1$ or ApoB $\geq 1.33 \mathrm{~g} / 1$, was diagnosed as hyperlipidemia.

DNA extraction. Venous blood $(2 \mathrm{ml})$ was collected into tubes following treatment with ethylenediaminetetraacetic acid and stored at $-80^{\circ} \mathrm{C}$. Genomic DNA was extracted using a DNA extraction kit (DNA purification kit; Omega Bio-Tek, Norcross, GA, USA) according to the manufacturer's instructions. DNA quantity and purity were measured using a spectrophotometer (BD 177742; BD Biosciences, Franklin Lakes, NJ, USA).

Genotyping. The p22phox gene was amplified by PCR using the following primers: Forward: 5'-GTGTGGCTGGAA TGGTGGCAGGAG-3' and reverse: 5'-GAGGGTCCCACC TGAGCCAATGTG-3' designed using primer 5 software (Premier Biosoft, Palo Alto, CA, USA). The primers were synthesized at Shanghai Shenggong Biological Engineering Co., Ltd. (Shanghai, China). PCR was performed using $25 \mu \mathrm{l}$ 2-fold PCR mixture, $1 \mu \mathrm{l}$ of each primer, $15 \mu \mathrm{l}$ template DNA in a $50 \mu \mathrm{l}$ final volume. The PCR amplification program was as follows: Initial denaturation at $95^{\circ} \mathrm{C}$ for $3 \mathrm{~min}$, denaturation at $95^{\circ} \mathrm{C}$ for $30 \mathrm{sec}$, annealing at $65.5^{\circ} \mathrm{C}$ for $30 \mathrm{sec}$, extension at $72^{\circ} \mathrm{C}$ for $45 \mathrm{sec}\left(35\right.$ cycles) and a final extension step at $72^{\circ} \mathrm{C}$ for $10 \mathrm{~min}$.

RFLP. The PCR products $(10 \mu \mathrm{l})$ were digested with

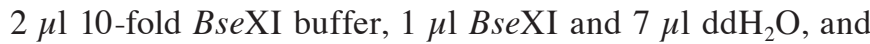
the mixture was incubated for $12 \mathrm{~h}$ at $65^{\circ} \mathrm{C}$. Following digestion, $4 \mu \mathrm{l}$ 6-fold DNA loading dye (Sigma-Aldrich, St. Louis, MO, USA) was added to the mixture. All products were separated by electrophoresis on a $2 \%$ agarose gel containing $0.1 \%$ ethidium bromide and were visualized under ultraviolet light. Digestion of the PCR products yielded bands of $254 \mathrm{bp}$ for the AA-homozygotes, 57 and 197 bp for the GG-homozygotes and three fragments in the AG-heterozygotes (Fig. 1). DNA purification was performed with a commercially available kit (QIAamp DNA kit; Qiagen, Duesseldorf, Germany) according to the manufacture's instruction. The PCR products of the three genotypes were randomly selected for DNA sequencing in an ABI 3130XL automated sequencer (Applied Biosystems, Foster City, CA, USA), according to the manufacturer's instructions, and were then sent for gene sequencing by Invitrogen Life Technologies (Carlsbad, CA, USA).

Statistical analysis. Statistical analysis was performed using SPSS 17.0 software (SPSS, Inc., Chicago, IL, USA). All quantitative data are expressed as the mean \pm standard error of the mean. Student's t-test was used to compare between two groups. The $\chi^{2}$ test was used to compare categorical variables. Deviation of the genotype and allele distribution from the Hardy-Weinberg equilibrium was measured using the $\chi^{2}$ test. $\mathrm{P}<0.05$ was considered to indicate a statistically significant difference. Binary logistic regression analysis was performed to estimate the independent risk factors for $\mathrm{ICH}$, which were identified when $\beta>0$, odds ratio $(\mathrm{OR})>1$ and $\mathrm{P}<0.05$.

\section{Results}

Subject characteristics. No significant differences were observed in gender, age or body mass index between the ICH 
Table I. Characteristics of intracerebral hemorrhage patients and healthy controls.

\begin{tabular}{|c|c|c|c|c|}
\hline Characteristic & Controls $(n=147)$ & Patients $(n=118)$ & F-value $/ \chi^{2}$ value & P-value \\
\hline Male, n (\%) & $88(59.9)$ & $70(59.3)$ & 0.008 & 0.929 \\
\hline Age (years) & $65.22 \pm 9.69$ & $66.28 \pm 14.53$ & 22.922 & 0.481 \\
\hline BMI $\left(\mathrm{kg} / \mathrm{m}^{2}\right)$ & $23.78 \pm 2.28$ & $23.64 \pm 2.62$ & 1.989 & 0.639 \\
\hline $\mathrm{SBP}(\mathrm{mmHg})$ & $135.29 \pm 19.67$ & $163.09 \pm 27.68$ & 19.269 & 0.000 \\
\hline $\mathrm{DBP}(\mathrm{mmHg})$ & $81.14 \pm 10.17$ & $92.67 \pm 16.08$ & 27.079 & 0.000 \\
\hline Blood glucose (mmol/l) & $6.01 \pm 2.14$ & $6.80 \pm 3.02$ & 8.494 & 0.015 \\
\hline Triglycerides (mmol/l) & $1.33 \pm 0.75$ & $1.73 \pm 1.07$ & 6.680 & 0.000 \\
\hline Cholesterol (mmol/l) & $4.59 \pm 0.98$ & $4.48 \pm 0.94$ & 0.019 & 0.037 \\
\hline HDL (mmol/l) & $1.34 \pm 0.34$ & $1.35 \pm 0.42$ & 5.181 & 0.886 \\
\hline LDL (mmol/l) & $3.00 \pm 0.88$ & $2.82 \pm 0.79$ & 0.676 & 0.036 \\
\hline $\operatorname{ApoA}(\mathrm{g} / \mathrm{l})$ & $1.39 \pm 0.27$ & $1.34 \pm 0.33$ & 5.693 & 0.204 \\
\hline ApoB (g/l) & $0.94 \pm 0.25$ & $0.88 \pm 0.26$ & 0.710 & 0.090 \\
\hline Smoker, n (\%) & $19(12.9)$ & $29(24.6)$ & 5.991 & 0.014 \\
\hline Alcohol drinker, n (\%) & $23(15.6)$ & $35(29.7)$ & 7.520 & 0.006 \\
\hline History of hypertension, n (\%) & $44(29.9)$ & $81(68.6)$ & 39.364 & 0.000 \\
\hline
\end{tabular}

Values are expressed as the mean \pm standard error of the mean. BMI, body mass index; SBP, systolic blood pressure; DBP, diastolic blood pressure; HDL, high density lipoprotein; LDL, low density lipoprotein; Apo, apolipoprotein.

Table II. Genotype and allele distributions for the patients with intracerebral hemorrhage and the healthy control group.

\begin{tabular}{lccc}
\hline Genotype/allele & Controls $(\mathrm{n}=147)$ & Patients $(\mathrm{n}=118)$ & $\chi^{2}$ value \\
\hline -A930G genotype, $\mathrm{n}(\%)$ & & & 12.828 \\
AA & $91(61.9)$ & $58(40.7)$ & 0.003 \\
AG & $43(29.3)$ & $16(13.6)$ & 10.645 \\
GG & $13(8.8)$ & & 0.001 \\
-A930G allele, n (\%) & & $150(63.6)$ & \\
A & $225(76.5)$ & $86(36.4)$ & \\
G & $69(23.5)$ & & \\
\hline
\end{tabular}

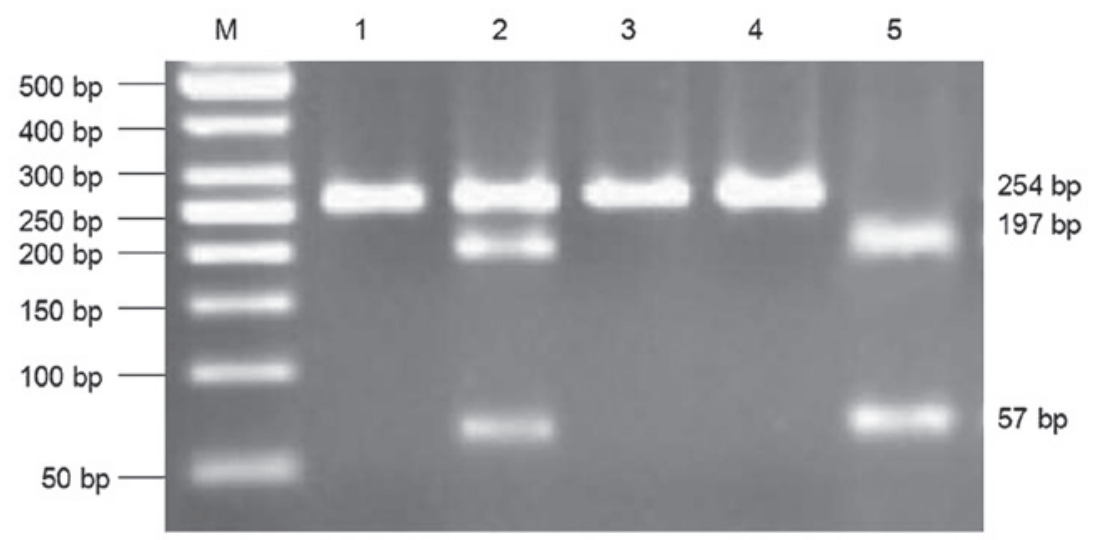

Figure 1. Genotyping of the -A930G polymorphism of the p22phox gene. M represents the marker; 1, 3 and 4 represent the AA genotype (254 bp); 2 represents the AG genotype (254, 197 and $57 \mathrm{bp}$ ) and 5 represents the GG genotype (197 and $57 \mathrm{bp}$ ).

and control groups $(\mathrm{P}>0.05)$, suggesting that the $\mathrm{ICH}$ group was comparable with the control group (Table I). The prevalence of conventional risk factors for ICH were significantly higher in the ICH group $(\mathrm{P}<0.05)$, which included systolic blood pressure (SBP), diastolic blood pressure (DBP), cholesterol, LDL, blood glucose, triglyceride, history of hypertension, smoking 
Table III. Intracerebral hemorrhage risk factors in binary logistic regression analysis.

\begin{tabular}{|c|c|c|c|c|c|}
\hline Risk factor & $\beta$-value & SEM & P-value & OR-value & $95 \% \mathrm{CI}$ \\
\hline Gender & -0.696 & 0.347 & 0.095 & 0.499 & $0.253-0.984$ \\
\hline SBP & 0.815 & 0.202 & 0.010 & 2.260 & $1.521-3.358$ \\
\hline DBP & 0.418 & 0.228 & 0.067 & 1.519 & $0.972-2.375$ \\
\hline Smoking & 1.078 & 0.444 & 0.015 & 2.938 & $1.229-7.021$ \\
\hline History of hypertension & 0.893 & 0.330 & 0.014 & 2.442 & $1.279-4.664$ \\
\hline Triglyceride level & 1.039 & 0.497 & 0.037 & 2.826 & $1.066-7.489$ \\
\hline Blood glucose & 1.172 & 0.425 & 0.006 & 3.228 & $1.403-7.426$ \\
\hline -A930G & 0.640 & 0.325 & 0.009 & 2.196 & $1.003-3.586$ \\
\hline
\end{tabular}

SEM, standard error of the mean; OR, odds ratio; CI, confidence interval; SBP, systolic blood pressure; DBP, diastolic blood pressure.

Table IV. Comparison between the clinical characteristics of different genotypes of the -A930G p22phox polymorphism.

\begin{tabular}{|c|c|c|c|c|c|}
\hline Clinical characteristic & AA (141) & AG (96) & GG (28) & F-value & P-value \\
\hline BMI $\left(\mathrm{kg} / \mathrm{m}^{2}\right)$ & $23.88 \pm 2.27$ & $23.48 \pm 2.69$ & $23.77 \pm 2.37$ & 0.770 & 0.464 \\
\hline $\mathrm{SBP}(\mathrm{mmHg})$ & $143.76 \pm 25.62$ & $151.60 \pm 27.56$ & $153.89 \pm 32.17$ & 3.225 & 0.041 \\
\hline DBP (mmHg) & $84.95 \pm 14.39$ & $88.41 \pm 14.00$ & $85.64 \pm 14.56$ & 1.706 & 0.184 \\
\hline Blood glucose (mmol/l) & $6.30 \pm 2.45$ & $6.66 \pm 3.07$ & $5.76 \pm 1.08$ & 1.438 & 0.239 \\
\hline Triglycerides (mmol/l) & $1.50 \pm 0.94$ & $1.52 \pm 0.86$ & $1.55 \pm 1.11$ & 0.045 & 0.956 \\
\hline Cholesterol (mmol/l) & $4.56 \pm 0.96$ & $4.58 \pm 0.99$ & $4.36 \pm 0.90$ & 0.613 & 0.542 \\
\hline HDL (mmol/l) & $1.32 \pm 0.36$ & $1.36 \pm 0.39$ & $1.39 \pm 0.43$ & 0.707 & 0.494 \\
\hline $\mathrm{LDL}(\mathrm{mmol} / \mathrm{l})$ & $2.93 \pm 0.83$ & $2.95 \pm 0.89$ & $2.71 \pm 0.79$ & 0.971 & 0.380 \\
\hline ApoA (g/l) & $1.36 \pm 0.28$ & $1.37 \pm 0.30$ & $1.35 \pm 0.34$ & 0.076 & 0.927 \\
\hline ApoB (g/l) & $0.92 \pm 0.27$ & $0.92 \pm 0.25$ & $0.86 \pm 0.21$ & 0.606 & 0.546 \\
\hline
\end{tabular}

Values are expressed as the mean \pm standard error of the mean. BMI, body mass index; SBP, systolic blood pressure; DBP, diastolic blood pressure; HDL, high density lipoprotien; LDL, low density lipoprotien; Apo, apolipoprotein.

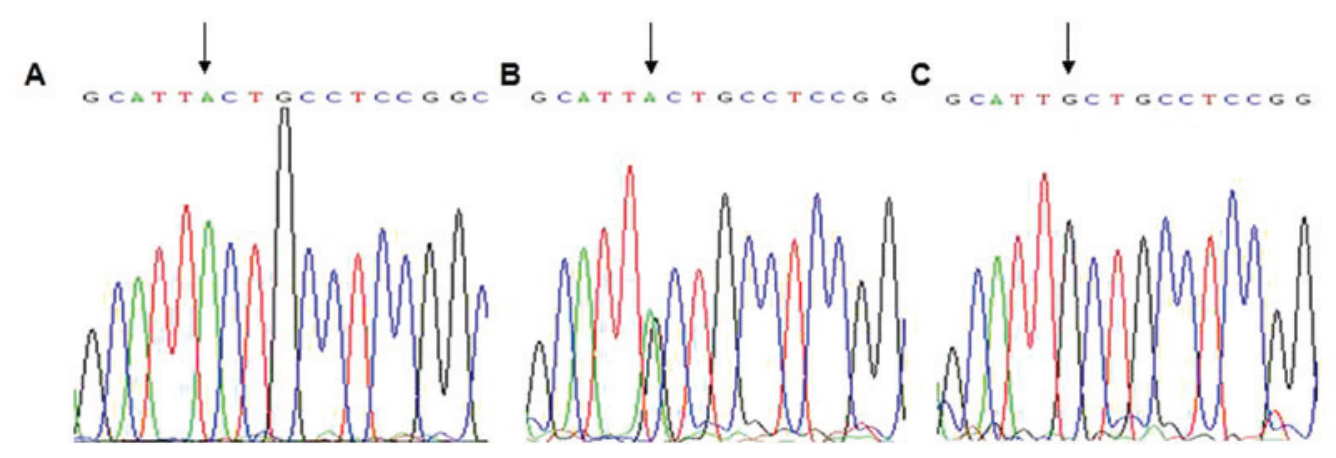

Figure 2. Genetic sequence of the -A930G polymorphism of the nicotinamide adenine dinucleotide phosphate oxidase p22phox subunit. (A) AA genotype; (B) AG genotype; (C) GG genotype. Arrows indicate a genetic polymorphism. Singe sequencing peaks represent homozygotes and double peaks refer to heterozygotes. Red peak, T; green peak, A; black peak, G; blue peak, C.

and alcohol consumption. No significant difference was observed in HDL, ApoA or ApoB between the patients and the controls $(\mathrm{P}>0.05)$.

Distribution of the -A930G polymorphism. The distribution of the -A930G polymorphism of the p22phox gene was accordance with the Hardy-Weinberg equilibrium in the $\mathrm{ICH}$ and control individuals. The PCR products were digested by $B s e X I$ at the 5'-GCAGCN8-3' site. The DNA of the AA genotype was not digested by the enzyme, yielding a 254 bp DNA fragment. The DNA of GG genotype was digested into two fragments of 57 and $197 \mathrm{bp}$. As only one chain of the AG gene was digested, the AG genotype DNA was cleaved into three fragments of 57, 197 and 254 bp (Fig. 1). DNA sequencing was performed with 
five PCR products of the AA, AG and GG genotypes according to the results of the restriction digestion (Fig. 2). The arrows indicate mutation sites, which were in accordance with the electrophoresis results. The distribution of p22phox -A930G genotypes demonstrated significant differences between the two groups (Table II, $\mathrm{P}=0.003$ ), with frequencies of the AA, AG, GG genotypes of $61.9,29.3$ and $8.8 \%$ in the controls, and $40.7,45.8$ and $13.6 \%$ in the ICH patients, respectively. The $\mathrm{G}$ allele frequency in the $\mathrm{ICH}$ patients was significantly higher compared with the control group, 36.4 and $23.5 \%$, respectively $(\mathrm{P}<0.05)$. By contrast, the frequency of the A allele was significantly lower in the ICH group compared with the controls, 63.6 and $76.5 \%$, respectively $(\mathrm{P}<0.05)$.

Binary logistic regression analysis of risk factors for ICH. Differences between the patients and controls were analyzed using binary logistic regression analysis, which revealed that the $-\mathrm{A} 930 \mathrm{G}$ polymorphism of the p22phox gene was an independent risk factor for $\mathrm{ICH}(\mathrm{P}=0.009$; OR, 2.196; 95\% CI, 1.003-3.586). However, SBP, DBP, blood glucose and triglyceride levels, and a history of hypertension and smoking may be associated with the risk of ICH (Table III).

Comparison of clinical data in different -A930G genotypes. Following comparison of the clinical data from the three -A930G genotypes, significant differences were observed in the level of SBP among the AA, AG and GG genotypes $(\mathrm{P}<0.05)$, while no significant differences were observed with the other parameters $(\mathrm{P}>0.05)$. These data suggested that genetic mutation of -A930G increased the level of SBP (Table IV).

\section{Discussion}

ROS are positively associated with cerebrovascular diseases. Enhanced ROS production at sites of injury regulates all the cellular responses to injury, including the induction of inflammatory genes, expression of adhesion molecules, monocyte adhesion and platelet aggregation (17). Alteration of the balance between endothelial proliferation and apoptosis by ROS causes excessive angiogenesis or loss of endothelial cells (18). ROS promote the activation, aggregation and adhesion of platelets on the endothelium at sites of thrombi and can cause mitochondrial dysfunction, leading to a reduction in the production of adenosine triphosphate and marked problems with energy metabolism (19). Evidence has revealed that p22phax is an essential component of the NAD(P)H oxidase system and is important in regulating the activity of $\mathrm{NAD}(\mathrm{P}) \mathrm{H}$ oxidases when producing ROS $(20,21)$.

The -A930G polymorphism of $\mathrm{NAD}(\mathrm{P}) \mathrm{H}$ p22phox is significantly associated with $\mathrm{NAD}(\mathrm{P}) \mathrm{H}$ oxidase activation and the production of ROS. San José et al (22) reported that hypertensive patients with the GG genotype have increased mRNA and protein levels of p22phox and higher NAD $(\mathrm{P}) \mathrm{H}$ oxidase activity compared with those with AA/AG genotypes, while no differences were observed between the GG and AA/AG genotypes in the healthy control group. Transfection experiments on vascular smooth muscle cells revealed that the $A$ to $G$ substitution increases the expression of the reporter gene in hypertensive cells and hypertensive subjects carrying the GG genotype of the p22phox
-A930G polymorphism are highly exposed to NAD(P)H oxidase-mediated oxidative stress (22). Golliasch et al (23) suggested that there is a protective association between the -A930G promoter polymorphisms in the NAD(P)H p22phox gene and the development of myocardial infarction in individuals $\leq 40$ years of age. Zalba et al (24) demonstrated the -A930G polymorphism as a novel marker in determining the genetic susceptibility of hypertensive patients to oxidative stress.

To the best of our knowledge, the present study demonstrated for the first time that the $-\mathrm{A} 930 \mathrm{G}$ polymorphism of the p22phox gene affects the susceptibility of the Chinese Han population to ICH. A significant difference was observed in the frequencies of the AA, AG, GG genotypes and A and $\mathrm{G}$ alleles between the $\mathrm{ICH}$ and control subjects. Logistic regression analysis indicated that genetic mutations of the p22phox -A930G gene were independent risk factors of ICH ( $\mathrm{P}=0.009$; OR, 2.196; 95\% CI, 1.003-3.586). The -A930G polymorphism of the p22phox gene was associated with the risk of developing ICH when the clinical data of different genotypes were included. One explanation for -A930G polymorphism as a potential risk factor of $\mathrm{ICH}$ is that the $\mathrm{G}$ allele was associated with higher blood pressure in the brachial artery and in the aorta (25). For this reason, the $-\mathrm{A} 930 \mathrm{G}$ polymorphism may have an indirect association with $\mathrm{ICH}$ by increasing blood pressure (26). Another possibility is that the $-\mathrm{A} 930 \mathrm{G}$ polymorphism is located at the promoter region of the p22phox gene and a $30 \%$ increase in promoter activity and increased oxidative stress was observed in the $G$ allele compared with the A allele (27), leading to endothelial dysfunction, thickening of the vessel wall and $\mathrm{ICH}$.

In conclusion, the present study demonstrated that the -A930G polymorphism of the NAD(P)H p22phox may be associated with $\mathrm{ICH}$ in the Chinese Han population and screening this site may aid the diagnosis of ICH patients in the future.

\section{Acknowledgements}

This study was supported by the Shanghai Songjiang Health Bureau, a leading cooperation class A foundation (grant no. 2011LX03).

\section{References}

1. Hua Y, Keep RF, Gu Y and Xi G: Thrombin and brain recovery after intracerebral hemorrhage. Stroke 40: S88-S89, 2009.

2. Dong XQ, Du Q, Yu WH, et al: Plasma resistin, associated with single nucleotide polymorphism -420 , is correlated with C-reactive protein in Chinese Han patients with spontaneous basal ganglia hemorrhage. Genet Mol Res 11: 1841-1850, 2012.

3. Khatri R, Tariq N, Vazquez G, et al: Outcomes after nontraumatic subarachnoid hemorrhage at hospitals offering angioplasty for cerebral vasospasm: a national level analysis in the United States. Neurocrit Care 15: 34-41, 2011

4. Aronowski J and Zhao X: Molecular pathophysiology of cerebral hemorrhage: secondary brain injury. Stroke 42: 1781-1786, 2011

5. Qureshi AI, Mendelow AD and Hanley DF: Intracerebral haemorrhage. Lancet 373: 1632-1644, 2009.

6. Kim H, Crago E, Kim M, et al: Cerebral vasospasm after sub-arachnoid hemorrhage as a clinical predictor and phenotype for genetic association study. Int J Stroke 8: 620-625, 2013.

7. Taniyama $\mathrm{Y}$ and Griendling KK: Reactive oxygen species in the vasculature: molecular and cellular mechanisms. Hypertension 42: 1075-1081, 2003. 
8. Li J, Zhou K, Meng X, et al: Increased ROS generation and SOD activity in heteroplasmic tissues of transmitochondrial mice with A3243G mitochondrial DNA mutation. Genet Mol Res 7: 1054-1062, 2008.

9. Cheng JC, Cheng HP, Tsai IC and Jiang MJ: ROS-mediated downregulation of MYPT1 in smooth muscle cells: a potential mechanism for the aberrant contractility in atherosclerosis. Lab Invest 93: 422-433, 2013.

10. Ng CK, Deshpande SS, Irani K and Alevriadou BR: Adhesion of flowing monocytes to hypoxia-reoxygenation-exposed endothelial cells: role of Rac1, ROS, and VCAM-1. Am J Physiol Cell Physiol 283: C93-C102, 2002.

11. Wang Y and Tabas I: Emerging roles of mitochondria ROS in atherosclerotic lesions: causation or association? J Atheroscler Thromb 21: 381-390, 2014.

12. Patel RP, Moellering D, Murphy-Ullrich J, Jo H, Beckman JS and Darley-Usmar VM: Cell signaling by reactive nitrogen and oxygen species in atherosclerosis. Free Radic Biol Med 28 1780-1794, 2000.

13. Qin YW, Peng J, Liang BY, et al: The A930G polymorphism of $\mathrm{P} 22$ phox (CYBA) gene but not C242T variation is associated with hypertension: a meta-analysis. PLoS One 8: e82465, 2013.

14. Muller G and Morawietz H: Nitric oxide, NAD(P)H oxidase, and atherosclerosis. Antioxid Redox Signal 11: 1711-1731, 2009.

15. Akasaki T, Ohya Y, Kuroda J, et al: Increased expression of gp91phox homologues of $\mathrm{NAD}(\mathrm{P}) \mathrm{H}$ oxidase in the aortic media during chronic hypertension: involvement of the renin-angiotensin system. Hypertens Res 29: 813-820, 2006.

16. Ushio-Fukai M, Zafari AM, Fukui T, Ishizaka $\mathrm{N}$ and Griendling KK: p22phox is a critical component of the superoxide-generating NADH/NADPH oxidase system and regulates angiotensin II-induced hypertrophy in vascular smooth muscle cells. J Biol Chem 271: 23317-23321, 1996.

17. Fraser PA: The role of free radical generation in increasing cerebrovascular permeability. Free Radic Biol Med 51: 967-977, 2011.

18. Papaharalambus CA and Griendling KK: Basic mechanisms of oxidative stress and reactive oxygen species in cardiovascular injury. Trends Cardiovasc Med 17: 48-54, 2007.
19. Seligmann C, Schimmer M, Leitsch T, et al: A thrombocyte-induced myocardial dysfunction in the ischemic and reperfused guinea pig heart is mediated by reactive oxygen species. Free Radic Biol Med 29: 1244-1251, 2000.

20. Collins-Underwood JR, Zhao W, Sharpe JG and Robbins ME: NADPH oxidase mediates radiation-induced oxidative stress in rat brain microvascular endothelial cells. Free Radic Biol Med 45: 929-938, 2008.

21. Ding $H$, Aljofan $M$ and Triggle CR: Oxidative stress and increased eNOS and NADPH oxidase expression in mouse microvessel endothelial cells. J Cell Physiol 212: 682-689, 2007.

22. San José G, Moreno MU, Oliván S, et al: Functional effect of the p22phox $-930 \mathrm{~A} / \mathrm{G}$ polymorphism on $\mathrm{p} 22$ phox expression and NADPH oxidase activity in hypertension. Hypertension 44: 163-169, 2004.

23. Goliasch G, Wiesbauer F, Grafl A, et al: The effect of p22-PHOX (CYBA) polymorphisms on premature coronary artery disease ( $\leq 40$ years of age). Thromb Haemost 105: 529-534, 2011

24. Zalba G, San José G, Moreno MU, Fortuño A and Díez J: Nadph oxidase-mediated oxidative stress: genetic studies of the p22(phox) gene in hypertension. Antioxid Redox Signal 7: $1327-1336,2005$.

25. Xaplanteris $\mathrm{P}$, Vlachopoulos $\mathrm{C}, \mathrm{Baou} \mathrm{K}$, et al: The effect of p22(phox) $-930 \mathrm{~A} / \mathrm{G}, \mathrm{A} 640 \mathrm{G}$ and $\mathrm{C} 242 \mathrm{~T}$ polymorphisms of NADPH oxidase on peripheral and central pressures in healthy, normotensive individuals. Hypertens Res 33: 814-818, 2010.

26. Moreno MU, San José G, Fortuño A, Beloqui O, Díez J and Zalba G: The C242T CYBA polymorphism of NADPH oxidase is associated with essential hypertension. J Hypertens 24: 1299-1306, 2006.

27. Fan M, Raitakari OT, Kähönen M, et al: The association between cigarette smoking and carotid intima-media thickness is influenced by the $-930 \mathrm{~A} / \mathrm{G}$ CYBA gene polymorphism: the Cardiovascular Risk in Young Finns Study. Am J Hypertens 22: 281-287, 2009. 